\title{
INVESTIGACIÓN
}

Recibido: 28/03/2019 --- Aceptado: 28/06/2019 --- Publicado: 15/09/2020

\section{DERECHOS POLÍTICOS DE LAS MUJERES EN UNA SOCIEDAD DEMOCRÁTICA}

\author{
Women's political rights in a democratic society
}

Sonia Escalante López: Universidad Autónoma de Sinaloa. México justicia.mc@hotmail.com

Rosa Isela Ávalos Méndez: Benemérita Universidad Autónoma de Puebla. México. isela.avalos@correo.buap.mx

\section{RESUMEN}

El presente trabajo aborda a través de la línea del tiempo de los derechos políticos de las mujeres en una sociedad democrática, para la realización del estudio, se hace un análisis de la lucha de las mujeres por sus derechos humanos a través de la historia, dando inicio con los postulados de Olympe de Gouges como punto de partida del feminismo en el mundo y la lucha por los derechos humanos de las mujeres.

PALABRAS CLAVE: Democracia - Derechos Humanos -Derechos Políticos, Mujeres.

\section{ABSTRACT}

This paper addresses, through a timeline, women's political rights in a democratic society. For the realization of this study, an analysis of the struggle of women for their human rights throughout history was conducted, beginning with the postulates of Olympe de Gouges as the starting point of feminism in the world and the fight for women's human rights.

KEY WORDS: Democracy - Human Rights - Political Rights, Women.

\section{DIREITOS POLÍTICOS DAS MULHERES EM UMA SOCIEDADE DEMOCRÁTICA}

\section{RESUMO}

O presente trabalho aborda através de uma linha de tempo os direitos políticos das mulheres em uma sociedade democrática, para a realização do estudo, se faz uma 
análise da luta das mulheres pelos seus direitos humanos através da história, iniciando com os postulados de Olympe de Gouges como ponto de partida do feminismo no mundo e a luta pelos direitos humanos das mulheres.

PALAVRAS CHAVE: Democracia - Direitos Humanos -Direitos Políticos, Mulheres.

\section{Como citar el artículo:}

Escalante López, S. y Ávalos Méndez, R. I. (2020). Derechos políticos de las mujeres en una sociedad democrática. [Women's political rights in a democratic society]. Vivat Academia. Revista de Comunicación, 152, 117-130.

doi: http:/ / doi.org/10.15178/va.2020.152.117-130 Recuperado de: http://www.vivatacademia.net/index.php/vivat/article/view/1270

\section{INTRODUCCIÓN}

Democracia una palabra con mucho significado siendo sus valores, la honestidad, la solidaridad, la responsabilidad, el pluralismo, la libertad, la justicia social, la tolerancia y la Igualdad. Pero, como lo señala Alexis de Toqueville (2015) en su obra "La Democracia en América" parafraseando a Platón pues si deseamos cambiar la vida ética de los hombres "El primer problema y el más urgente es encontrar el verdadero orden político, encontrando este, podrá procederse a la formación de los hombres buenos que a su vez contribuirán a la formación del estado justo", esto alude a ese orden político debe prevalecer en cualquier democracia y a los hombres justo en un estado de derecho.

La democracia como forma de gobierno creada por el hombre ha sido la mejor a través de la historia desde su génesis en la antigua Grecia en el siglo VI antes de cristo, y por su parte muchos pensadores han llevado los ideales de la verdadera democracia, como Abraham Lincoln que expresaba la esencia de las palabras griegas, del sentido de la democracia, el gobierno del pueblo, por el pueblo y para el pueblo.

Es precisamente que en esas asambleas del pueblo en Grecia los ciudadanos eran libres para votar en la creación de leyes, con excepción de los esclavos y las mujeres. $Y$ en esa forma de gobierno político que defiende la soberanía del pueblo y el derecho del pueblo a elegir y controlar a sus gobernantes, se encuentran vinculado los derechos humanos, en la época contemporánea y con la evolución de derecho y la internacionalización del derecho procesal de los derechos humanos, se ha fortalecido la democracia en el mundo.

\section{OBJETIVOS}

El objetivo eje del presente artículo de investigación fue analizar el avance que se ha tenido en el corpus iuris de los derechos de las mujeres, hasta llegar al estudio de las sentencias de la Corte Interamericana de Derechos Humanos y los postulados que 
describen en sus sentencias sobre la importancia y salvaguarda de los derechos políticos en la democracia en las américas.

\section{METODOLOGÍA}

Este trabajo aborda el estudio de los derechos políticos de las mujeres en una sociedad democrática, para ello se realiza desde un enfoque cualitativo estudios comparados sobre el derecho a votar y ser votado de las mujeres, a través de los métodos analítico, histórico y síntesis.

\section{DISCUSIÓN}

\subsection{La Democracia y el Sistema Interamericano}

Con la evolución de los derechos humanos nace la Carta Democrática Interamericana, aprobada en la primera sesión plenaria del 11 de septiembre de 2001 de la Asamblea General de la Organización de los Estados Americanos, en donde queda plasmado que en el Sistema Interamericano existe la relación entre derechos humanos, democracia representativa y los derechos políticos en particular, describiendo en el artículo 3 de la Carta democrática (OEA, 2011): El objetivo de la carta democracia es con el fin de: lograr la estabilidad, la paz y el desarrollo de la región siendo uno de los propósitos de la Organización de Estados Americanos, el de promover y consolidar la democracia representativa dentro del respeto del principio de no intervención; pero que además reconoce los aportes de la OEA y de otros mecanismos regionales y subregionales en la promoción y consolidación de la democracia en las América.

Con ello reafirma que la participación de la democracia en nuestros países en los diferentes ámbitos de la actividad pública contribuye a la consolidación de los valores democráticos, como, la libertad, la solidaridad, igualdad, legalidad, justicia social, pluralismo y responsabilidad.

En el caso del Sistema Interamericano, los estados partes de este, tienen la obligación de promoverla y defenderla para que sus gobernados tenga la libertad de participación permanente bajo el principio de legalidad, considerando que en un sistema democrático se fortalece el desarrollo social económico y político, además, del robustecimiento de los partidos politos y candidaturas independientes, bajo la supervisión de la transparencia y rendición de cuentas.

En este mismo contexto, la Corte Interamericana de Derechos Humanos, ha señalado en el Caso Yatama Vs. Nicaragua que "en una sociedad democrática los derechos y libertades inherentes a la persona, sus garantías y el Estado de Derecho constituyen una tríada", en la que cada componente se define, completa y adquiere sentido en función de los otros dos. Al ponderar la importancia que tienen los derechos políticos la Corte observa que incluso la Convención, en su artículo 27, 
prohíbe su suspensión y la de las garantías judiciales indispensables para la protección de éstos.

2. La disposición precedente no autoriza la suspensión de los derechos determinados en los siguientes artículos: 3 (Derecho al Reconocimiento de la Personalidad Jurídica); 4 (Derecho a la Vida); 5 (Derecho a la Integridad Personal); 6 (Prohibición de la Esclavitud y Servidumbre); 9 (Principio de Legalidad y de Retroactividad); 12 (Libertad de Conciencia y de Religión); 17 (Protección a la Familia); 18 (Derecho al Nombre); 19 (Derechos del Niño); 20 (Derecho a la Nacionalidad), y 23 (Derechos Políticos), ni de las garantías judiciales indispensables para la protección de tales derechos.

\subsection{Los Derechos Políticos de las Mujeres}

En esta investigación cuando se hace referencia a los derechos de las mujeres no debemos olvidar y reconocer el legado de Olympe de Gouges, una revolucionaria olvidada, una mujer que se involucró activamente en la lucha por la igualdad, aun en esos tiempos en donde las mujeres no tenían prácticamente derechos de participación, propuso programas de reformas sociales dirigido a las tres primeras legislaturas de la Revolución, su trabajo adquirió un aspecto feminista y revolucionario, defendió la igualdad entre el hombre y la mujer en todos los ámbitos de la vida pública y privada, incluyendo el derecho al voto, el acceso al trabajo público y a la vida política, el derecho a poseer y controlar las propiedades, a la educación, etc.

En 1791 redactó la Declaración de los Derechos de la Mujer y la Ciudadana, fundándose en la declaración de los Derechos del Hombre y del Ciudadano, el cual presentó al parlamento, sin embargo, el 1783 fue guillotinada, durante mucho tiempo estuvo en el olvido fue finales de segunda guerra mundial, que Olympe de Gouges se consideró como una de las grandes figuras humanistas de Francia, una de sus frases, "si la mujer puede subir al caldazo, también deberían de reconocer su derecho a subir a tribuna".

Hombre, ¿Eres capaz de ser justo? Una mujer te hace esta pregunta, por lo menos no le prives de ese derecho.

Dime, ¿qué te da imperio soberano para oprimir a mi sexo? ¿Tu fuerza? ¿Tus talentos? Observa al Creador en su sabiduría, observa en toda su grandiosidad esa naturaleza con la cual parece que quieres estar en armonía, y dame, si te atreves, un ejemplo de su imperio tiránico.

En esta parte del preámbulo de la Declaración de los Derechos de la Mujer y de la Ciudadana, Olympe de Gouges, expresa que la mujeres tienen derechos naturales inalienables y pregunta al hombre quien le ha dado ese poder tiránico, considerando este punto de partida como la discriminación entre ambos.

Si hacemos un recuento desde la declaración de Virgina de 1776 señalando la búsqueda de la felicidad del hombre, el artículo $1^{\circ}$. De la Declaración de los 
Derechos del Hombre y del Ciudadano de 1789, describe: los hombres nacen libres en igualdad de derechos, por lo que se advierte que este documento estaba destinado a los hombres y a una clase social, como la burguesía, en el caso de la declaración de los Derechos de la Mujer y de la Ciudadana, es el inicio de la línea del tiempo de la defensa de los derechos de las mujeres, en el caso propio no me extra pronunció atacando a los hombres, porque creo que todo tiene su equilibrio y si se busca la igualdad, en principio debe de prevalecer el respeto, máxime que somos engendrados por un varón, tenemos padre, hermanos, hijos, sobrinos, amigos que son hombres, etc., no es una pelea de género, es la búsqueda de la igualdad en todos los ámbitos de la vida pública y privada.

Artículo 1. Que todos los hombres son por naturaleza igualmente libres e independientes y tienen ciertos derechos innatos, de los cuales, cuando entran en un estado de sociedad no se les puede privar, por un pacto, en sus posteridad; es decir, el disfrute dela vida y la libertad, con los medios de adquirir y poseer propiedades y de buscar y obtener felicidad y seguridad. (Declaración de los Derechos de Virginia de 1776).

Artículo 1. Los hombres nacen y viven libres e iguales en derechos, Las distinciones sociales solo pueden ser fundadas en la utilidad común. Declaración de los Derechos del Hombre y del Ciudadano 1789.

Es precisamente en ese caminar de la lucha de las mujeres a través de la historia que se han dado grandes acontecimientos como es el caso de lograr votar y ser votada, en la evolución de las sociedades modernas podemos decir que se han alcanzado importantes avances de reconocimiento y goce de los derechos humanos de las mujeres.

En la línea del tiempo de la lucha de las mujeres por el derecho al voto, las hojas de la historia marca que la primera colonia en reconocer el derecho al sufragio femenino universal fue en 1893 en nueva Zelanda, (Revista Socio Cultural, Lo ojos de Hipatia) https://losojosdehipatia.com.es/seguida de Australia Merdirional en 1894, el primer país que reconoció el derecho de postulación a cargos públicos y sufragios fue Finlandia en 1906, las primeras Filandesas fueron elegidas para ocupar el parlamento de 1907.

Ahora, para 1910 con la efervescencia que se vivía por la revolución mexicana, había una gran participación de las mujeres como las Adelita, otras mujeres que han quedado en el olvido, pero, también hay que reconocer que la historia les da vida, además un grupo de mujeres sufragistas que se denominaban "Las hijas de Cuauhtémoc, (La Silla Rota) https://lasillarota.com/lacaderadeeva/las-hijas-decuauhtemoc-el-movimiento-feminista-de-la-revolucion-aniversario/337208 apoyaron a Francisco I Madero en su lucha anti reeleccionista y protestaban por la participación femenina en la vida política del país, y fue por ello que en 1916 se celebró el Primer Congreso Feminista que acordó el voto ciudadano de las mujeres, no obstante, fue omitido en la constitución de 1917, pero en ese año en el Estado de Yucatán se logra que se otorgue la ciudadanía para votar a las mujeres mayores de 
21 años y en 1923 Elvia Carrillo Puerto, resulta electa como la primera diputada al Congreso Local por el V Distrito de Yucatán, y en ese camino de la historia las mujeres, pasaron muchos lustros de luchas y en un mitin el 6 de abril de 1952 que reunió a más de 20 mil mujeres en el parque 18 de marzo de la ciudad de México, en donde demandaban el derecho al voto, y fue en 1953 por mandato presidencial Adolfo Ruiz Cortines promulgó la reforma al artículo 34 y 115 fracción I de la Constitución Política de los Estados Unidos Mexicanos y es así que el 3 de julio de 1955 las mujeres por primera vez emitieron su voto para elegir diputados federales.

Artículo 34. Son ciudadanos de la República los varones y las mujeres que, teniendo la calidad de mexicanos, reúnan, además, los siguientes requisitos:

1-Haber cumplido 18 años, siendo casados, o 21 si no lo son; y

11.-Tener un modo honesto de vivir. (DOF. de 7 de octubre de 1953).

Todavía en la Declaración Americana de los Derechos y Deberes del Hombre de 1948 describe:

Todos los hombres nacen libres e iguales en dignidad y derechos y dotados como están por naturaleza de razón y conciencia, deben conducirse fraternamente como unos con los otros.

En el avance del reconocimiento y goce de los derechos humanos la Declaración Universal de los Derechos Humanos, en esta declaración ya no describe el hombre sino todos los seres humanos, incluso es el primer documento que señala la palabra derechos humanos, con esto abre la puerta para considerar que las mujeres son personas humanas.

Artículo 1. Todos los seres humanos nacen libres e iguales en dignidad y derecho y, dotados como están de razón y conciencia, deben de comportarse fraternalmente los unos con los otros.

A través de la historia se ha mostrado como la mujer ha sido segregada, mancillada ultrajada, y haciendo memoria en el pasaje del libro de la Suerte de la Consorte de Sara Sefchovich, narra a las esposas de los Presidentes de México, aquellas que sólo tenían derecho a cuidar a sus hijos, y hacer chocolates, en esa obra Sara describe el caso en 1884 de Laura Mantecón de González, mujer sola y sin recursos harta de los ultrajes y humillaciones de su marido, se atrevió a pelear en contra del poderío Manuel del Refugio González Flores, Presidente de los Estados Unidos Mexicanos, este valiéndose de su poder reformó el Código Civil, para despojarla de todos los bienes y de sus hijos “El cónyuge que diere causa al divorcio perderá todo su poder y derechos sobre la persona y bienes de sus hijos y perderá todo lo que se le hubiere dado o prometido por su consorte".

Ha sido un vaivén de las mujeres en México, las sentencias de la Corte Interamericana de Derechos Humanos ha dado pauta a que en México se realicen reformas de gran trascendencia en materia de derechos humanos como la del 10 de junio de 2011, la cual cimbro al sistema jurídico en nuestro país, constitucionalmente 
se pasó de un reconocimiento al goce de los derechos humanos y en esa medida en el artículo 1 párrafo segundo establece: Las normas relativas a los derechos humanos se interpretarán de conformidad con esta Constitución y con los tratados internacionales de la materia favoreciendo en todo tiempo a las personas la protección más amplia.

Este párrafo señala la interpretación conforme de la constitución y los tratados internacionales en materia de derechos, además, de establecer el principio pro persona, que quiere decir que siempre se otorgara lo que más beneficie a la persona, también, que en rango constitucional control difuso de convencionalidad, en esta misma vertiente en su párrafo tercero ordena lo siguiente:

Todas las autoridades, en el ámbito de sus competencias, tienen la obligación de promover, respetar, proteger y garantizar los derechos humanos de conformidad con los principios de universalidad, interdependencia, indivisibilidad y progresividad. En consecuencia, el Estado deberá prevenir, investigar, sancionar y reparar las violaciones a los derechos humanos, en los términos que establezca la ley.

En este ordenamiento describe la obligatoriedad de todas las autoridades para promover respetar, proteger y garantiza los derechos humanos, bajo los principios de universalidad, interdependencia, indivisibilidad y progresividad, es decir todos los derechos humanos para todos los humanos, los derechos humanos no pueden dividirse y dependen uno de otros, pero además, siempre se protegerán progresivamente salvaguardando cada uno de ellos, para evitar la re victimización, o la repetición de violación a derechos humanos.

A pesar de que en nuestra Constitución Política de los Estados Unidos, establecía que el hombre y la mujer son iguales ante la ley, se logra la paridad de género y el 6 de junio de 2019 se publica en Diario Oficial de la Federación la reforma a los artículos 2, 4, 41, 52, 53, 56, 94 y 115 de la Constitución Política de los Estados Unidos Mexicanos, sobre paridad de género, con ello las mujeres logran una participación equilibrada de hombres y mujeres en las posiciones de poder y de toma de decisiones en todas las esferas de la vida (políticas, económicas y sociales), constituye una condición destacada para la igualdad entre los sexos, de tal manera que el grado de paridad de las instituciones políticas y económicas se considera actualmente un indicador de la calidad democrática de los países, integrándose este dato en numerosos índices internacionales.

Considerando que el equilibrio de hombres y mujeres demanda que se refleje mejor la construcción de la sociedad, y que se garanticen los intereses de las mujeres en la elaboración de las políticas públicas y que se elimine la percepción que la política es cosa de hombres, tal y como lo señalan los artículos 35 y 41:

Artículo 35. Son derechos de la ciudadanía: II. Poder ser votada en condiciones de paridad para todos los cargos de elección popular, teniendo las calidades que establezca la ley. El derecho de solicitar el registro de candidatos y candidatas ante la autoridad electoral corresponde a los partidos 
políticos, así como a los ciudadanos y las ciudadanas que soliciten su registro de manera independiente y cumplan con los requisitos, condiciones y términos que determine la legislación.

Artículo 41. La ley determinará las formas y modalidades que correspondan, para observar el principio de paridad de género en los nombramientos de las personas titulares de las secretarías de despacho del Poder Ejecutivo Federal y sus equivalentes en las entidades federativas. En la integración de los organismos autónomos se observará el mismo principio. (DOF.6/6/2019).

Con esta reforma se caen esos techos de cristal que tanto han servido de muro de contención limitando el paso a la igualdad sustantiva de las mujeres, con ello se cierra la brecha y florece la igualdad de oportunidades para las mujeres y hombres, en un régimen democrático como México existe un cuerpo de derecho que posibilita la participación de todas las personas en la toma de decisiones y acceso a las oportunidades en la administración pública y privada, en este avance de la lucha de los derechos de las mujeres existe también un gran corpus iuris sobre los derechos de las mujeres, que permite la garantía de la participación política y en cualquier otro ámbito.

Como se ha señalado la internacionalización del derecho constitucional y el derecho procesal de los derechos humanos ha fortalecido la democracia en américa, en el caso de los derechos políticos la Corte interamericana de Derechos Humanos se ha pronunciado el Tratado en forma detallada la eficacia del artículo 23 de la Convención Americana sobre Derechos Humanos, sobre el rol de los derechos políticos en una sociedad democrática, el contenido y transcendencia de los derechos políticos, la obligatoriedad del Estado para promoverlo respetarlos y garantizarlos en su máxima expresión en igualdad de condiciones, en relación al contenido del artículo 23 de la Convención Americana sobre Derechos Humanos la Corte Interamericana se ha pronunciado sobre el tema de los derechos políticos en los siguientes casos:

\section{1) Caso Yatana vs Nicaragua 23 de junio de 2005}

El caso Yatana fue uno de los primeros casos en que la Corte Interamericana de Derechos Humanos conoce de los derechos políticos:

191. La Corte ha establecido que "en una sociedad democrática los derechos y libertades inherentes a la persona, sus garantías y el Estado de Derecho constituyen una tríada", en la que cada componente se define, completa y adquiere sentido en función de los otros. Al ponderar la importancia que tienen los derechos políticos la Corte observa que incluso la Convención, en su artículo 27, prohíbe su suspensión y la de las garantías judiciales indispensables para la protección de éstos.

192. Este Tribunal ha expresado que "la democracia representativa es determinante en todo el sistema del que la Convención forma parte", y 
constituye "un 'principio' reafirmado por los Estados americanos en la Carta de la OEA, instrumento fundamental del Sistema Interamericano". Los derechos políticos protegidos en la Convención Americana, así como en diversos instrumentos internacionales, propician el fortalecimiento de la democracia y el pluralismo político.

\section{2) Castañeda Gutman Vs. México. Sentencia del 6 de agosto de 2008}

140. Los derechos políticos son derechos humanos de importancia fundamental dentro del sistema interamericano que se relacionan estrechamente con otros derechos consagrados en la Convención Americana como la libertad de expresión, la libertad de reunión y la libertad de asociación y que, en conjunto, hacen posible el juego democrático. La Corte destaca la importancia que tienen los derechos políticos y recuerda que la Convención Americana, en su artículo 27, prohíbe su suspensión y la de las garantías judiciales indispensables para la protección de éstos.

141. Los derechos políticos consagrados en la Convención Americana, así como en diversos instrumentos internacionales, propician el fortalecimiento de la democracia y el pluralismo político. Este Tribunal ha expresado que "la democracia representativa es determinante en todo el sistema del que la Convención forma parte", y constituye "un 'principio' reafirmado por los Estados americanos en la Carta de la OEA, instrumento fundamental del Sistema Interamericano".

144. El artículo 23.1 de la Convención establece que todos los ciudadanos deben gozar de los siguientes derechos y oportunidades, los cuales deben ser garantizados por el Estado en condiciones de igualdad: i) a la participación en la dirección de los asuntos públicos, directamente o por representantes libremente elegidos; ii) a votar y a ser elegido en elecciones periódicas auténticas, realizadas por sufragio universal e igual y por voto secreto que garantice la libre expresión de los electores; y iii) a acceder a las funciones públicas de su país.

94. El artículo 23 de la Convención consagra los derechos a la participación en la dirección de los asuntos públicos, a votar, a ser elegido, y a acceder a las funciones públicas, los cuales deben ser garantizados por el Estado en condiciones de igualdad.

\section{3) Caso López Mendoza Vs. Venezuela. Del 1 de septiembre de 2011}

196. La participación política puede incluir amplias y diversas actividades que las personas realizan individualmente $\mathrm{u}$ organizados, con el propósito de intervenir en la designación de quienes gobernarán un Estado o se encargarán de la dirección de los asuntos públicos, así como influir en la formación de la política estatal a través de mecanismos de participación directa.

197. El ejercicio de los derechos a ser elegido y a votar, íntimamente ligados 
entre sí, es la expresión de las dimensiones individual y social de la participación política.

198. Los ciudadanos tienen el derecho de participar en la dirección de los asuntos públicos por medio de representantes libremente elegidos. El derecho al voto es uno de los elementos esenciales para la existencia de la democracia y una de las formas en que los ciudadanos ejercen el derecho a la participación política.

En estos casos que mencionamos la Corte IAD ha considerado el soporte del artículo 23 de la Convención $\mathrm{ADH}$ en el caso de los derechos políticos:

1. Todos los ciudadanos deben gozar de los siguientes derechos y oportunidades:

a) de participar en la dirección de los asuntos públicos, directamente o por medio de representantes libremente elegidos;

b) de votar y ser elegidos en elecciones periódicas auténticas, realizadas por sufragio universal e igual y por voto secreto que garantice la libre expresión de la voluntad de los electores; y

c) de tener acceso, en condiciones generales de igualdad, a las funciones públicas de su país.

2. La ley puede reglamentar el ejercicio de los derechos y oportunidades a que se refiere el inciso anterior, exclusivamente por razones de edad, nacionalidad, residencia, idioma, instrucción, capacidad civil o mental, o condena, por juez competente, en proceso penal.

Incluso la Corte IAD consideró que este artículo fue desarrollado en el artículo 2 de la Carta Democrática Interamericana de 2001: "La democracia representativa se refuerza y profundiza con la participación permanente, ética y responsable de la ciudadanía en un marco de legalidad conforme al respectivo orden constitucional"

Como lo hemos subrayado con anterioridad la evolución del derecho y de los derechos humanos ha fortalecido la participación política de las personas en todo el continente americano, en el caso de los derechos políticos de las mujeres, durante el transcurso del tiempo se ha ido cerrando la brecha de la desigualdad de oportunidades incluso la propia Corte Interamericana a considerado.

4) Caso López Lone y otros Vs. Honduras de 5 de octubre de 2015

149. Como punto de partida cabe resaltar que la democracia representativa es uno de los pilares de todo el sistema del que la Convención forma parte y constituye un principio reafirmado por los Estados americanos en la Carta de la OEA, instrumento fundamental del sistema interamericano. En este sentido, la Carta de la OEA, tratado constitutivo de la organización de la cual Honduras es Parte desde el 7 de febrero de 1950, establece como uno de sus propósitos esenciales "la promoción y la consolidación de la democracia 
Escalante López, S. y Ávalos Méndez, R. I.

Derechos politicos de las mujeres en una sociedad democrática

representativa dentro del respeto al principio de no intervención"

5) Corte IDH. Caso San Miguel Sosa y otras Vs. Venezuela. de 8 de febrero de 2018

110. El artículo 1.1 de la Convención es una norma de carácter general, cuyo contenido se extiende a todas las disposiciones del tratado, ya que dispone la obligación de los Estados Parte de respetar y garantizar el pleno y libre ejercicio de los derechos y libertades en ella reconocidos, "sin discriminación alguna". Es decir, cualquiera sea el origen o la forma que asuma, todo tratamiento que pueda ser considerado discriminatorio respecto del ejercicio de cualquiera de los derechos garantizados en la Convención es incompatible con la misma.

111. El artículo 23 de la Convención Americana contiene diversas normas que se refieren a los derechos de la persona como titular del proceso de toma de decisiones en los asuntos públicos, como elector a través del voto o como servidor público, es decir, a ser elegido popularmente o mediante designación o nombramiento para ocupar un cargo público.

En virtud de esta disposición, las personas también tienen "el derecho de participar activamente en la dirección de los asuntos públicos directamente, mediante referendos, plebiscitos o consultas o bien, por medio de representantes libremente elegidos". A diferencia de casi todos los demás derechos previstos en la Convención que se reconocen a toda persona, el artículo 23 de la misma no solo establece que sus titulares gozan de derechos, sino que agrega el término "oportunidades", lo cual implica la obligación del Estado de garantizar con medidas positivas y de generar las condiciones y mecanismos óptimos para que toda persona formalmente titular de esos derechos tenga la oportunidad real para ejercerlos, de forma efectiva, respetando el principio de igualdad y no discriminación. En este sentido, es necesaria la existencia de institucionalidad y mecanismos de carácter procedimental que permitan y aseguren el efectivo ejercicio del derecho, previniendo o contrarrestando situaciones o prácticas legales o de facto que impliquen formas de estigmatización, discriminación o represalias para quien lo ejerce.

En la sentencia del caso Yana contra Perú del 23 de junio de 2005 en su párrafo 94 describe que el artículo 23 de la Convención $\mathrm{ADH}$ debe de ser interpretado en conexión con el derecho a la igualdad que se encuentra establecido en el artículo 24 de la propia convención y el artículo 7 que describe que los derechos humanos son indivisibles e interdependientes. La Corte IDH ha considerado que la noción de la igualdad emana de la naturaleza del género humano. Considerando además que el principio de igualdad concierne al us cogens, amparando de esta manera los derechos colectivos.

Por su parte la Suprema Corte de Justicia de la Nación se ha pronunciado señalando que las discusiones en torno a los derechos fundamentales a la igualdad y la no discriminación suelen transitar por tres ejes. 
1. La necesidad de adoptar ajustes razonables para lograr una igualdad sustantiva y no meramente formal entre las personas

2. La adopción de medidas especiales o afirmativas, normalmente llamadas acciones afirmativas

3. Análisis de actos y preceptos normativos que directa o indirectamente por resultado o de forma tácita sean discriminatorios (tesis 44/2018) $10^{\mathrm{a}}$.

Hemos señalado como ha evolucionado el derecho y los derechos humanos incluyendo los derechos políticos de las mujeres en los sistemas democráticos, y valdría la pena preguntarse porque los régimen democráticos, en ocasiones pasan a hacer dictaduras, para ello Steven Leviski y Danielk Xiblatt, en el libro cómo mueren las democracias, señalan que a pesar de que los gobernantes llegan al gobierno de forma democrática es decir el pueblo le otorga la soberanía, estos a su vez se enamoran del poder y se vuelven tiranos, es de esta forma como mueren las democracias.

En la obra de Noam Chomsky "Quien Domina al Mundo" señala que en el caso de Estados Unidos estudios han demostrado que la gran mayoría de la población en el extremo bajo de la escala de ingreso riqueza, se halla de hecho excluida del sistema político y sus opiniones y posturas son pasadas por alto por sus representantes formales, mientras que en un pequeño sector en la cima posee una influencia arrolladora, en ambas posturas interpreto que al final de todo y a pesar de la evolución del derecho orientado a la garantía de los derechos humanos, incluyendo los políticos, siempre estará el poderoso, el que limita la participación de los pobres y mata la democracia, cuanto ha cambiado desde aquellos postulados de la antigua Grecia.

\section{CONCLUSIONES}

En este estudio hemos advertido al desarrollo que han tenido los derechos humanos, en el caso los derechos políticos de las mujeres en una sociedad democrática, el fortalecimiento del cuerpo normativo para la garantía de los derechos humanos en igualdad de participación en la toma de decisiones, en su obra la Republica de Platón, menciona que un gobernante que no incluye a las mujeres en su gabinete es una hombre que tiene cabeza pero no tiene brazos, desde entonces platón vislumbraba a las mujeres en los gabinetes gobernantes, sin embargo, a través de las páginas de la historia en la búsqueda de los derechos de las mujeres no ha sido fácil llegar a la toma de decisiones, aun teniendo un estado democrático, no obstante, el progreso de los derechos humanos las sentencias de la Corte Interamericana de Derechos Humanos, las resoluciones y tesis de la Suprema Corte de Justicia de la Nación Mexicana, ha dado garantía a las mujeres para obtener los derechos humanos, los derechos políticos en igualdad de condiciones. 
Escalante López, S. y Ávalos Méndez, R. I.

Derechos politicos de las mujeres en una sociedad democrática

\section{REFERENCIAS}

Carta Democrática Interamericana, aprobada en la primera sesión plenaria, 11 de septiembre de 2001 de la Asamblea General de la Organización de los Estados Americanos.

Chomsky, N. (2016). ¿Quién Domina el Mundo? Barcelona: Ediciones BSA.

Cienfuegos Salgado, D. (2005). La historia de los Derechos Humanos. México Distrito Federal: Comisión de Defensa de los Derechos Humanos.

Constitución Política de los Estados Unidos Mexicanos.

Declaración Americana de los Derechos y Deberes del Hombre, 1948.

Declaración de los Derechos de la Mujer y de la Ciudadana, 1793.

Declaración de los Derechos del Hombre y del Ciudadano, 1793.

Declaración de Virginia, 1776.

Diario Oficial, 6 de julio de 2019-

Diario Oficial, 7 de octubre de 1953.

Levitski, S.; Daniel, Z (2018). Cómo Mueren las Democracias. México Distrito Federal Ariel

Platón (1992). La República. México Distrito Federal: Editores Mexicanos Unidos

Redacción. (2019). Las hijas de Cuauhtemoc, el movimiento feminista de la revolución. Recuperado de https://lasillarota.com/lacaderadeeva/las-hijas-de-cuauhtemocel-movimiento-feminista-de-la-revolucion-aniversario/337208

Sentencia de la Corte Interamericana de Derechos Humano. Castañeda Gutman Vs. México, 6 de agosto de 2008.

Sentencia de la Corte Interamericana de Derechos Humanos, Caso Yatana vs Nicaragua, 23 de junio de 2005.

Sentencia de la Corte Interamericana de Derechos Humanos. Caso López Mendoza Vs. Venezuela, 1 de septiembre de 2011

Sentencia de la Corte Interamericana de Derechos Humanos. Caso López Lone y otros Vs. Honduras, 5 de octubre de 2015. 
Sentencia de la Corte Interamericana de Derechos Humanos. Caso San Miguel Sosa y otras Vs. Venezuela, 8 de febrero de 2018.

Setchovit, S. (2002). La Suerte de la Consorte. Las esposas de los gobernantes de México Historia de un olvido y relato de un fracaso. México Distrito Federal: Océano.

Steiner, Ch.; Uribe P. (2014). Convención Americana de Derechos Humanos, Comentada, México Distrito Federal: SCJN.

Toqueville, A. (2008). La Democracia en América, México Distrito Federal: Fondo de Cultura Económica.

\section{AUTOR/ES:}

\section{Sonia Escalante López}

Doctora en Derecho, con especialidad en derecho procesal constitucional y garantías constitucionales, Licenciatura en Derecho, Doctorado Honoris Causa, por la Universidad Bonagens, campus Aguas Calientes,Honorary Jus Doctor, por la Universidad American International school of law. Participación en las Audiencias Públicas y el Seminario "El sistema interamericano de Derechos Humanos, tendencias y complementariedades", Medellín, Colombia, 2013, Corte Interamericana de Derechos Humanos, Autora de 3 libros y coautora en 23 libros, en donde destacan. La independencia del Poder Judicial en México, Justicia Constitucional, Hacia su desarrollo en Sinaloa, Los Derechos Humanos en la Seguridad Pública y la Función Policial, Argumentación Jurídica, Legislativa y Jurisdiccional, y Derecho Procesal Convencional y la Inconvencionalidad, autora 20 artículos en revistas arbitradas e indexadas, Directora y fundadora de la revista digital arbitrada Derecho\&opinionciudadana. Actualmente Directora del Instituto de Investigaciones Parlamentarias del Congreso de Sinaloa México. Correo electrónico, justicia.mc@hotmail.com.

Orcid ID: https://orcid.org/0000-0002-2089-0050

\section{Rosa Isela Ávalos Méndez}

Es abogada por la Facultad de Derecho Y Ciencias Sociales de la Benemérita Universidad Autónoma de Puebla (BUAP), Maestra en Derecho con Terminal en Derecho Civil y Mercantil, Doctorante en Ciencias de Gobierno y Política, ProfesoraInvestigadora de la BUAP, Miembro de la Barra de Abogados de Puebla, A.C. Abogada General de Benemérita Universidad Autónoma de Puebla de 2015 a la fecha. isela.avalos@correo.buap.mx 